A N N A L E S Annales de Bretagne et des Pays de l'Ouest

Anjou. Maine. Poitou-Charente. Touraine

124-3 | 2017

Accueil et soin de l'enfant

\title{
Le hochet d'Archytas : un jouet pour grandir
}

Archytas's rattle: a toy with which to grow up

\section{Véronique Dasen}

\section{OpenEdition}

\section{Journals}

Édition électronique

URL : http://journals.openedition.org/abpo/3697

DOI : $10.4000 / a b p o .3697$

ISBN : 978-2-7535-6539-5

ISSN : 2108-6443

\section{Éditeur}

Presses universitaires de Rennes

Édition imprimée

Date de publication : 16 novembre 2017

Pagination : 89-107

ISBN : 978-2-7535-5674-4

ISSN : 0399-0826

\section{Référence électronique}

Véronique Dasen, «Le hochet d'Archytas : un jouet pour grandir », Annales de Bretagne et des Pays de I'Ouest [En ligne], 124-3 | 2017, mis en ligne le 16 novembre 2019, consulté le 03 janvier 2020. URL : http://journals.openedition.org/abpo/3697 ; DOI : 10.4000/abpo.3697 


\title{
Le hochet d'Archytas : un jouet pour grandir ${ }^{1}$
}

\author{
Véronique DASEN \\ Professeure d'archéologie classique, université de Fribourg, \\ membre associé de l'UMR 8210 ANHIMA, Paris
}

Sur la panse d'un cruchon ou chous attique à figures rouges, un jeune enfant est représenté nu, de face, assis dans un pot surélevé qui lui sert de chaise percée (fig. 1 ; vers $440-425$ av. J.-C.) $)^{2}$. Il remue ses petites jambes, disposées de manière asymétrique dans l'ouverture du siège pour suggérer leur agitation, tout en brandissant dans la main droite le manche d'un objet de forme circulaire, rehaussé de blanc, qui semble être un hochet. Deux objets posés par terre l'entourent, à sa gauche un bâton à roulettes, ou hamaxis, appuyé contre le cadre du panneau peint, à sa droite un petit vase, un chous semblable au récipient qui sert de support à l'image. D'ordinaire, la scène est interprétée comme l'évocation d'un moment de détente dans l'intimité du gynécée, dévoilant le monde récréatif de l'enfant grec. Cependant, cette lecture plaque un concept moderne sur une image antique. L'amalgame est dû à l'apparente banalité du hochet qui reçoit presque toujours le même bref commentaire, répété d'un livre à l'autre, le réduisant à un simple jouet distrayant. Un examen plus approfondi des éléments qui composent la scène révèle que l'imagier a construit un discours visuel élaboré sur le processus d'anthropopoiêsis qui transforme par étapes le tout-petit en être social.

1. Sauf mention contraire, toutes les traductions sont celles de la CUF (Collection des Universités de France) avec unification des différents termes utilisés en français pour traduire platagê.

2. Londres, British Museum, 1910.6-15.4; BA 11041; vAN HooRn, Gerard, Choes and Anthesteria, Leyde, Brill, 1951, p. 148, n 665. Le vase est souvent reproduit dans les ouvrages sur la vie quotidienne en Grèce ancienne. Par exemple FiTTÀ, Marco, Spiele und Spielzeug in der Antike, Stuttgart, Konrad Theiss, 1998, p. 75, fig. 136; NEILS, Jennifer, OAKLEY, John (dir.), Coming of Age in Ancient Greece : Images of Childhood from the Classical Past, New Haven/Londres, Yale Univ. Press/Hanover N.H./Hood Museum of Art/Dartmouth College, 2003, p. 240, n 41 ; BEAumont, Leslie A., Childhood in Ancient Athens. Iconography and Social History, Londres, Routledge, 2013, p. 58-59, fig. 3.11. 


\section{Des mots pour le dire}

Dans l'Antiquité grecque et romaine, le hochet est désigné comme un idiophone $^{3}$, un instrument dont le son est produit par le matériau de l'objet lui-même. Le mot le plus usité, ì $\pi \lambda \alpha \tau \alpha \gamma \eta$, souvent traduit en français par " hochet ", " crécelle " ou " cliquette ${ }^{4}$ ", est associé au verbe $\pi \lambda \alpha \tau \alpha \gamma \varepsilon ́ \omega$, " frapper des mains ". La notice platagê de la Souda (fin $\mathrm{x}^{\mathrm{e}}$ siècle) se réfère au son d'un grelot enfermé à l'intérieur d'un objet : le hochet y est défini comme " une sorte d'instrument qui produit une résonance, êkhos, et un son, psophos ${ }^{5}$ ".

Dans la Politique, Aristote donne au jouet une fonction au premier regard très prosaïque, occuper l'enfant pour qu'il ne sème pas le désordre dans la maison :

" Il faut considérer comme une belle invention le hochet, platagê, d'Archytas, que l'on donne aux petits enfants pour que, grâce à elle, ils ne cassent rien dans la maison, car la gent enfantine n'est pas capable de rester tranquille ${ }^{6}$."

Aristote ne décrit pas l'aspect de l'objet, mais il lui attribue un inventeur, une figure historique célèbre, Archytas de Tarente (435/410-360/350 av. J.-C.) ${ }^{7}$. Cette affirmation surprend pour au moins deux raisons. D'une part, les hochets sont attestés archéologiquement bien avant le $\mathrm{v}^{\mathrm{e}}$ siècle av. J.-C. ${ }^{8 ;}$ si ce type d'objet était connu depuis longtemps, pourquoi vouloir fixer son apparition chronologiquement en l'associant à un inventeur? D'autre part, Archytas est un homme politique d'envergure ${ }^{9}$, élu sept fois stratège de la cité de Tarente, commandant militaire, également philosophe pythagoricien, ami de Platon, d'une réputation éminente qui contraste avec la trivialité de l'objet auquel Aristote l'associe. L'identité de l'inven-

3. Dournon, Geneviève, "Instruments de musique du monde : foisonnement et systématiques. Essai de classification organologique ", dans NATTIEZ, Jean-Jacques (dir.), Musiques. Une encyclopédie pour le XX⿸厃 siècle, 5, L'unité de la musique, Paris/Arles, Cité de la Musique/Actes Sud, 2007, p. 843-849.

4. Les termes français utilisés par les traducteurs ne désignent cependant pas le même type d'idiophone. Dans la classification de Dournon, Geneviève, "Instruments de musique du monde... ", art. cité, la cliquette (ou crotale) est un idiophone par entrechoc de deux éléments semblables, la crécelle un idiophone par raclement, avec une roue dentée, le hochet un idiophone par secouement. Je remercie Florence Gétreau de cette remarque.

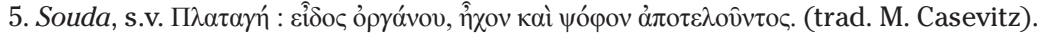

6. ArISTOTE, Politique, VIII, 6, 2, $1340 \mathrm{~b}$.

7. Sur les questions de chronologie, Huffman, Carl A., Archytas of Tarentum, Pythagorean, Philosopher and Mathematician King, Cambridge, Cambridge University Press, 2005, p. 5-6.

8. Par ex. Smith, Christopher J., "Dead Dogs and Rattles : Time, Space and Ritual Sacrifice in Iron Age Latium ", dans Wilkins, John B. (dir.), Approaches to the Study of Ritual : Italy and the Ancient Mediterranean, Londres, Accordia, p. 73-90 (Latium, vIII ${ }^{\mathrm{e}}$ siècle av. J.-C., tombe d'un enfant de trois ans avec un hochet sphérique en métal); BELLIA, Angela, Strumenti musicali e oggetti sonori nell'Italia meridionale e in Sicilia (VI-III sec. a. C.). Funzioni rituali e contesti, Lucca, Libreria Musicale Italiana, 2012, p. 14-34.

9. Mathieu, Bernard, "Archytas de Tarente, pythagoricien et ami de Platon ", Bulletin de l'Association Guillaume Budé, 1987, 1, p. 239-255. 
teur diffère cependant dans une variante rapportée par le grammairien Diogenianos à l'époque d'Hadrien. Archytas serait un charpentier, tektôn ${ }^{10}$ :

"Hochet d'Archytas : désigne tous ceux qui ne sont pas capables de se tenir tranquilles. Car Archytas, un charpentier, inventa le hochet, platagê, que l'on donne aux enfants, paidia, pour qu'ils ne cassent pas tout dans la maison." "

Comme Carl A. Huffman le relève, l'auteur a peut-être jugé nécessaire de préciser que l'inventeur devait être un artisan, et non le célèbre philosophe ${ }^{11}$, d'autant plus que la profession de charpentier est adaptée à la fabrication de hochets en bois. La notice de la Souda termine en indiquant l'existence de l'expression "le hochet d'Archytas ", qui témoigne de la valeur exemplaire de l'invention ${ }^{12}$.

Dans l'Onomasticon de Pollux de Naucratis (II ${ }^{\mathrm{e}}$ siècle apr. J.-C.) ${ }^{13}$, l'idiophone est désigné par le terme platagonion. Il est utilisé comme synonyme de krotalon, "crotale ", et de seistron, "sistre ", deux autres types d'instruments idiophones, l'un, krotalon, par entrechoc de deux éléments en bois ou en métal, l'autre, seistron, par secouement d'éléments enfilés sur des tiges en métal :

" [Platagonion]. On appelle ainsi la crotale, krotalon, et le sistre, seistron, avec quoi les nourrices conduisent au sommeil, en les charmant avec des berceuses, les enfants, paidia, qui ont du mal à dormir ${ }^{14}$."

Pour le lexicographe, cette famille de sons est aussi apparentée au bruit sec produit en faisant claquer une feuille ou un pétale de fleur posé sur l'anneau formé par le pouce et l'index afin de connaître la force des sentiments de l'être aimé ${ }^{15}$.

En latin également, deux termes suggestifs désignent le hochet comme un instrument idiophone, crepitaculum et crepitacillum, dérivés de crepare, " rendre un son sec, craquer, claquer, retentir ", et de crepitare, "émettre un bruit sec régulier ${ }^{16}$ ". Le mot sistrum peut aussi le décrire. Dans l'épigramme

10. Corpus paroemiographorum graecorum, I, 213 (Diogenianos II, 98,1); HufFMAN, Carl A., Archytas of Tarentum..., op. cit., texte A, p. 302-307.

11. Ibidem, p. 303.

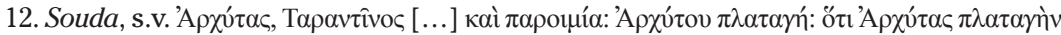

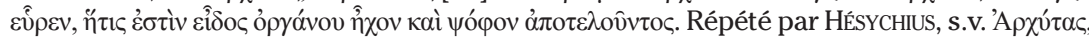

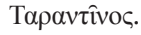

13. Pollux, Onomasticon, IX, 127.

14. Trad. M. Casevitz.

15. Deux mots désignent ce jeu divinatoire : "Les amoureux des deux sexes jouaient au platagonion, "craque-pétale" [...]. En enroulant autour des deux premiers doigts de la main gauche les feuilles de la plante appelée têlephilon et en formant ainsi un cercle, on frappe dessus avec le plat de l'autre main, et si la feuille, fendue par le coup, rend un bruit sonore, on pense que les amoureux se souviennent l'un de l'autre. " Sur ce jeu, Costanza, Salvatore, "Il “m'ama non m'ama” con il telephilon ", Maia, 65, 2013, p. 559-573.

16. Cf. Plaute, Rudens, 536 : " claquer des dents ", crepito dentibus; Properce, Élégies, $3,10,4$ : " applaudir ", crepuere manibus. Crepundia appartient à la même famille, mais 
intitulée crepitacillum, " le hochet ", Martial invite ainsi à distraire un enfant triste avec le son d'un sistre ${ }^{17}$ :

«Crepitacillum. Si un jeune esclave se suspend à ton cou en pleurant, qu'il agite ce sistre bavard, garrula sistra, de sa petite main. "

À l'inverse, dans les Métamorphoses, Apulée utilise le terme crepitaculum pour décrire un sistre métallique ${ }^{18}$ :

"Quant aux attributs de la déesse, ils étaient fort divers. Sa main droite portait un crepitaculum de bronze, aereum, dont le cadre étroit, recourbé en forme de baudrier, était traversé de quelques petites tiges qui, sous la triple secousse du bras, rendaient un son clair."

\section{Un hochet pour grandir}

La fonction de l'objet correspond à la conception qu'ont les Anciens du corps et des besoins de l'enfant. Elle révèle l'extrême attention portée dès la naissance aux étapes de son développement physiologique et psychique. La première phase de croissance débute avec un état d'apparente insensibilité pendant les quarante premiers jours ${ }^{19}$. Pour Aristote, les longues périodes de sommeil que traverse le nouveau-né sont associées à son entrée progressive dans le monde des vivants :

"Le passage du non-être à l'être s'effectue par l'état intermédiaire : or le sommeil semble bien être par nature un état de ce genre. Il est pour ainsi dire aux confins de la vie et de l'absence de vie, et le dormeur paraît ni complètement ne pas exister, ni exister ${ }^{20}$."

Cette étape de transition est jugée dangereuse, comme toute période de changement :

"La plupart des décès de petits enfants se produisent avant le septième jour. C'est pourquoi les enfants ne reçoivent leur nom que le septième jour, parce qu'on pense qu'ils ont désormais plus de chance de survivre ${ }^{21}$."

désigne les breloques qui servent d'amulettes; Plaute, Le soldat fanfaron, 1399 : quasi puero in collo pendeant crepundia.

17. MARTIAL, Épigrammes, XIV, 54 : Si quis plorator collo tibi vernula pendet, Haec quatiat tenera garrula sistra manu.

18. APULÉE, Métamorphoses, XI, 4 (trad. rev. par F. Gétreau) : Nam dextra quidem ferebat aereum crepitaculum, cuius per angustam lamminam in modum baltei recuruatam traiectae mediae paucae uirgulae, crispante brachio trigeminos iactus, reddebant argutum sonorem.

19. Hippocrate, Du foetus de 8 mois, 9,8 (Littré VII, 450) : « Ils ne rient ni ne pleurent spontanément avant quarante jours. Ils ne rient même pas avant ce même laps de temps si on les touche ou les chatouille : leurs facultés sont émoussées par les mucosités. "

20. ARISTOTE, Génération des animaux, v, 1, 778b. Voir aussi Aristote, Génération des animaux, v, 1, 779a : "Les enfants au début, restent endormis plus de temps que les autres animaux."

21. ARISTOTE, Histoire des animaux, VII, 12, 588a. Dans la médecine humorale hippocratique, tous les changements sont dangereux car ils créent un déséquilibre. 
Quand la quarantaine de jours est écoulée, émerge un être qui est à l'inverse dominé par les sensations et les émotions, immodérément, sans contrôle, semblable à un petit animal. Ce tempérament excessif résulte de sa nature chaude et humide dans la tradition hippocratique, sanguine chez Aristote $^{22}$. L'enfant devient alors sensible à son environnement, visuel et sonore $^{23}$. Des cris et des pleurs constituent son premier mode d'expression, géré de différentes manières ${ }^{24}$. Platon juge normales ces manifestations bruyantes car elles font partie du processus de développement :

" Tout nouveau-né s'exprime d'ordinaire par des cris, et tout particulièrement celui de race humaine, lequel d'ailleurs non content de crier, est plus que les autres enclin à pleurer ${ }^{25}$."

Il recommande cependant d'éviter de laisser pleurer trop longtemps les petits et incite à tenter de déchiffrer ce premier langage, comme le font avec succès les nourrices qui déploient une grande ingéniosité :

"Aussi les nourrices, en quête de ses désirs, usent-elles de ces indices pour deviner ce qu'elles doivent lui offrir : ce dont l'offre le fait taire, elles ont, concluent-elles, raison de l'offrir; ce qui le fait crier et pleurer, de ne pas l'offrir. Les enfants ont donc, pour manifester ce qu'ils aiment ou haïssent, leurs larmes, leurs plaintes, infortuné langage. Cette période ne dure pas moins de trois ans, portion de vie non minime à vivre bonne ou mauvaise ${ }^{26}$."

À l'inverse, Aristote considère que les pleurs sont utiles et représentent une sorte d'exercice pour le souffle, propre à rendre vigoureux :

«Les grands cris des enfants et leurs pleurs bruyants, c'est à tort que les interdisent ceux qui les prohibent dans leurs lois, car ils sont utiles pour la croissance; c'est en quelque sorte une gymnastique pour le corps : retenir son souffle donne de la force à qui fait des travaux pénibles et c'est aussi le cas des petits enfants dans de telles tensions ${ }^{27}$."

L'avis dominant est toutefois de les éviter à cause de la fragilité du corps enfantin. Des pleurs prolongés pourraient faire du mal, comme l'explique Soranos d'Éphèse (II ${ }^{\mathrm{e}}$ siècle apr. J.-C.) :

"Cela lui fait du bien parfois de pleurer : c'est un exercice naturel pour renforcer le souffle et les organes respiratoires et comme les voies s'élar-

22. Hippocrate, Du régime, I, 33 (Littré VI, 510-513). PsEudo-Aristote, Problèmes, I, 19, 861b. Répété par GALIEN, Hygiène, 1, 7 (Kühn 6, 31-37). Voir les sources rassemblées par HANSON, Ann Ellis, "Your Mother Nursed you with Bile": Anger in Babies and Small Children ", dans Braund, Susanna, Most, Glenn W. (dir.), Ancient Anger : Perspectives from Homer to Galen, Cambridge, Cambridge University Press, p. 185-207.

23. Hippocrate, Du foetus de 8 mois, 9, 8 (Littré viI, 450) : "S'ils échappent aux quarante jours, plus forts et plus intelligents en effet ils voient plus clairement la lumière et entendent le bruit, chose qu'ils ne pouvaient faire auparavant. "

24. Cf. Delaisi de Parseval, Geneviève, Lallemand, Suzanne, L'art d'accommoder les bébés, 100 ans de recettes françaises de puériculture, Paris, Seuil, 1980.

25. Platon, Lois, VII, 791e.

26. Ibidem, 791a-792a.

27. ARISTOTE, Politique, vII, 17, 6, 1336a. À Sparte, cependant, selon Plutarque, les nourrices doivent habituer les enfants à se retenir de pleurer, Vie de Lycurgue, 16, 4. 
gissent en se dilatant, la répartition de la nourriture est rendue plus aisée. Il ne faut cependant pas laisser l'enfant pleurer longtemps, car cela lui abîme les yeux et provoque la descente des intestins dans le scrotum ${ }^{28}$."

Les pleurs sont aussi redoutés car ils peuvent être le symptôme d'un accès de frayeur, phobos, qui constitue une des principales menaces pour la santé de l'enfant. Une forte émotion peut en effet causer des maux graves ${ }^{29}$. Un aphorisme hippocratique inscrit ces peurs dans une énumération d'affections en apparence banales, comme les aphtes, mais potentiellement fatales pour le tout petit qui ne peut plus s'alimenter normalement :

"Voici ce qui arrive selon les âges : chez les enfants, paidia, petits, smikra, et nouveau-nés, neogna, des aphtes, des vomissements, des toux, des insomnies, des terreurs, phoboi, des inflammations de l'ombilic, des suintements d'oreille ${ }^{30}$."

Dans le traité De la maladie sacrée, ces terreurs peuvent entraîner une crise épileptique $^{31}$ :

"Une cause non apparente peut encore la produire, par exemple une crainte, phobos, si l'enfant a peur de quelqu'un qui crie, ou encore l'impossibilité de reprendre promptement haleine dans l'intervalle de cris et pleurs, ce qui arrive souvent à cet âge. "

La crainte de la nocivité des peurs enfantines constitue un topos qui traverse la littérature antique, grecque et romaine ${ }^{32}$. Une frayeur vive est tenue pour responsable du déclenchement d'une crise épileptique, désignée par l'expression "maladie d'enfant ", paidion nosêma ${ }^{33}$. Différentes mesures sont préconisées pour les éviter, à commencer par s'abstenir de raconter des histoires effrayantes pour impressionner et faire obéir l'enfant. Platon

28. Soranos, Des maladies des femmes, II, 13. Rufus d'Éphèse (II ${ }^{\mathrm{e}}$ siècle apr. J.-C.) aurait également préconisé les pleurs des enfants comme un exercice et un moyen d'expulser salive et mucus; cité par Oribase, Livres incertains, 20 (Dar. III, 160). Sur la pédiatrie à l'époque romaine, BERTIER, Janine, "La médecine des enfants à l'époque impériale ", $A N R W$, II, 37, 3, 1996, p. 2147-2227; Hummel, Christine, Das Kind und seine Krankheiten in der griechischen Medizin. Von Aretaios bis Johannes Aktuarios (1. bis 14. Jahrhundert), Frankfurt am Main etc., 1999; MudRY, Philippe, "Non pueri sicut uiri. Petit aperçu de pédiatrie romaine ", dans DASEN, Véronique (dir.), Naissance et petite enfance dans l'Antiquité romaine, Fribourg/Göttingen, Academic Press/Vandenbroeck \& Ruprecht, 2004, p. 451-462.

29. Voir aussi CELSE, De la médecine, 10, 11. Les frayeurs sont aussi dangereuses pour la femme enceinte car elles peuvent causer un avortement; HIPPOCRATE, Maladies des femmes, I, 25 (Littré VIII, 66).

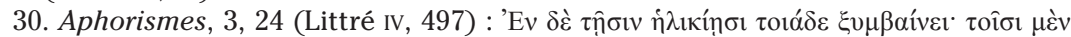

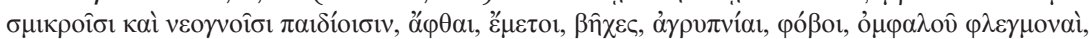

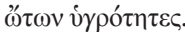

31. HiPPOCRATE, De la maladie sacrée, 10 (Littré VI, 378-381).

32. PAtera, Maria, Figures grecques de l'épouvante de l'antiquité au présent, Peurs enfantines et adultes (Mnemosyne suppl. 376), Leiden, Brill, 2014.

33. RuFus D'ÉPHÈSE, cité par ORIBASE, Livres incertains, 20 (Dar. III, 160-161). L'expression se trouve déjà chez HIPPOCRATE, Airs, eaux, lieux, 3 (Littré II 18), mais sans référence aux frayeurs. Sur cette maladie, voir dans ce volume GaIllard-SEuX, Patricia, " L'épilepsie de l'enfant dans l'Antiquité ( $\mathrm{I}^{\mathrm{er}}-\mathrm{V}^{\mathrm{e}}$ siècles) : prévention et traitement ". 
déconseille vivement de les menacer avec des mormolukeia ou phobêtra, des épouvantails qui rendent l'enfant craintif ${ }^{34}$ :

"Que de leur côté les mères n'aillent pas, sur la foi des poètes, effrayer leurs jeunes enfants en leur contant mal à propos que des dieux circulent pendant la nuit, déguisés en étranger sous mille formes diverses, et qu'ainsi elles évitent à la fois de blasphémer contre les dieux et de rendre leurs enfants plus peureux ${ }^{35}$."

La recommandation est répétée à l'époque romaine par Soranos qui ajoute que la peur peut engendrer des maux physiques aussi ${ }^{36}$ :

" [La nourrice] jamais ne l'effraiera ou ne l'inquiétera par des bruits en prenant un ton menaçant : la frayeur qui résulte de ces pratiques occasionne des maladies aussi bien physiques que mentales. "

Le souci est toujours présent chez Basile de Césarée (IV ${ }^{e}$ siècle apr. J.-C.) :

« Ne crois pas que je sois comme une mère ou une nourrice, brandissant devant toi de faux épouvantails, mormolukeia, comme elles ont coutume de faire avec les plus jeunes des enfants; lorsque ceux-ci, indisciplinés et impossibles à arrêter, se lamentent, elles les font taire au moyen de récits mensongers ${ }^{37}$."

L'équilibre de l'enfant peut être retrouvé grâce au sommeil réparateur que lui procurent le balancement, le chant doux des berceuses et le son régulier du hochet. Dans les Lois de Platon, ces soins doivent être prodigués de manière continue pour le bien du corps, $\sigma \hat{\omega} \mu \alpha$, et de l'âme, $\psi v \chi \eta ́$ :

" Alimentation et mouvement autant que possible ininterrompus de nuit comme de jour, voilà ce qui profite à tous, mais spécialement aux tout petits qui devraient, s'il se pouvait, vivre sans cesse bercés comme dans un navire; voilà, pour les tout nouveaux nourrissons, l'idéal qu'il faut essayer de réaliser au plus près ${ }^{38}$."

Il précise l'effet attendu du balancement et des berceuses en utilisant le verbe $\kappa \alpha \tau \alpha v \lambda \varepsilon ́ \omega$ qui évoque un pouvoir similaire à celui d'une incantation ${ }^{39}$ :

34. Hésychius, s.v. Mombrô et Mommô; PATERA Maria, Figures grecques de l'épouvante..., op. cit., p. 118.

35. Platon, République, II, 381a, 2-6.

36. SorAnos, Maladies des femmes, II, 13. Voir aussi Rufus D'ÉPHÈsE, cité par ORIBASE, Livres incertains, 20 (Dar. III, 160-161) : « On évitera aussi, plus que toute autre chose, la frayeur, les grands bruits et les cris à l'oreille, et on n'essayera pas de les effrayer subitement par l'apparition de spectres, ou de quelque autre vision. "

37. BASILE de CÉSARÉE, Homilia dicta tempore famis et siccitatis, Patrologia graeca, éd. J.-P. Migne, vol. 31, Paris, 1857, col. 328, 27-31; PATERA, Maria, Figures grecques de l'épouvante..., op. cit., p. 306-307.

38. Platon, Lois, VII, 790c-e. Relevons que Platon, Lois, VII, 789e-790a préconise de bercer l'enfant dans le ventre déjà pour le former : « La femme enceinte se promènera : elle modèlera ( $\pi \lambda \alpha$ á $\tau \varepsilon \imath v)$ son nouveau-né comme une cire tant qu'il est tendre. "

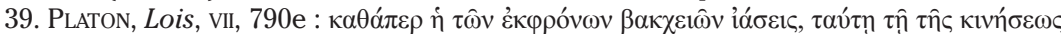

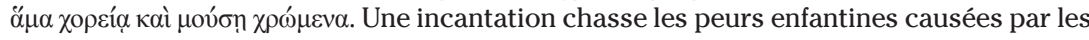
mormolukeia ; Platon, Phédon, 77e, 3-7. 
"Quand les mères, en effet, veulent endormir des enfants qui ont le sommeil difficile, au lieu de repos, c'est, au contraire, du mouvement, qu'elles leur donnent en les balançant sans cesse dans leurs bras, et, au lieu du silence, quelque chantonnement : disons que, au plein sens du mot, elles

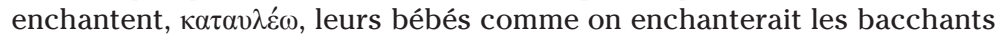

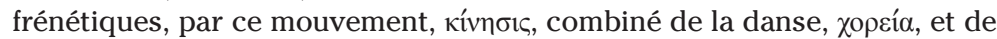
la musique, $\mu$ ov̂ $\sigma \alpha^{40}$."

Les auteurs de l'époque romaine ajoutent parfois des détails qui évoquent l'univers matériel enfantin, mais sans juger utile de donner d'autres précisions. Rufus d'Éphèse propose ainsi de rassurer l'enfant effrayé " en lui montrant les objets qui lui sont habituellement les plus chers ", tout en l'embrassant et en chantant " une de ces chansons de nourrice " pour l'endormir en le berçant " car, par l'effet du sommeil, il revient à lui et oublie sa peur, phobos ${ }^{41}$ ".

Le contenu de ces berceuses, $\beta \alpha v \kappa \alpha \lambda \eta ́ n \alpha \tau \alpha$ ou vóvvi $\alpha$, est presque entièrement perdu, à l'exception de brèves citations qui donnent une idée du genre, à défaut de reproduire les berceuses réelles. Théocrite fait ainsi chanter Alcmène pour endormir Héraclès et Iphiclès. Comme Andromache Karanika le relève, l'usage de l'impératif assure le caractère performatif de la berceuse qui doit procurer un sommeil apaisant, garantissant le réveil des petits au matin ${ }^{42}$. La douceur de cette scène donne plus de relief au caractère dramatique de l'épisode qui suit, l'attaque des serpents envoyés par Héra ${ }^{43}$. Théocrite précise les gestes que la mère accomplit en berçant les petits :

«Et, de sa main de femme caressant la tête des enfants, elle dit : "Dormez, mes petits, d'un sommeil doux et dont on se réveille. Dormez, mes chères âmes, les deux frères, les beaux enfants. Heureux puissiez-vous reposer, heureux d'atteindre l'aurore." Parlant ainsi, elle agita le grand bouclier; et le sommeil les saisit ${ }^{44}$."

Le souci culturel d'éviter que l'enfant soit saisi de peurs se retrouve dans les amulettes montées en breloque, alliant la puissance de la forme, du matériau et parfois aussi du son. Pline l'Ancien rapporte ainsi que " la dent de loup en amulette chasse les frayeurs des enfants et les maladies de

40. Pollux, Onomasticon, Ix, 127, utilise aussi le verbe psychagogousai pour désigner le pouvoir calmant du chant des berceuses.

41. Cité par ORIBASE, Livres incertains, 20 (Dar. III, 160-161).

42. Sur les berceuses grecques, WÆRN, Ingrid, " Greek Lullabies », Eranos, 58, 1960, p. 1-8; KARANIKA, Andromache, Voices at Work. Women, Performance, and Labor in Ancient Greece, Baltimore, Maryland, Johns Hopkins University Press, 2014, p. 160-164. Sur la proximité du sommeil et de la mort, Pseudo-Plutarque, Résumé des opinions des philosophes, 24, Euvres morales, 909e-f.

43. Le contexte narratif des berceuses est souvent associé à un moment de danger;

PACHE, Corinne O., Baby and Child Heroes in Ancient Greece, Urbana and Chicago, Illinois Press, 2004, p. 107-112.

44. THÉOCRITE, Idylle, XXIV, 6-10. 
la dentition, comme le fait aussi la peau du loup ${ }^{45}$ ". De même, une dent de dauphin portée en amulette " chasse les frayeurs soudaines ${ }^{46}$ ".

Le son du hochet lui-même aurait-il eu le pouvoir d'éloigner les forces nuisibles invisibles qui menacent les enfants? Des chercheurs ont parfois avancé l'hypothèse qu'il pouvait écarter les démons, comme le faisaient les hochets du Moyen Âge qui renfermaient sept grains symbolisant les sept péchés capitaux, sans donner plus de détails ${ }^{47}$. Cette fonction est envi-

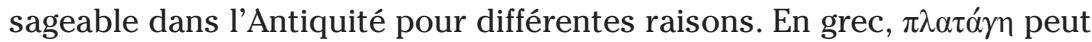
désigner un instrument en métal dont le bruit écarte de manière efficace les êtres malfaisants. Dans les Argonautiques d'Apollonios de Rhodes (III ${ }^{\mathrm{e}}$ siècle av. J.-C.), Héraclès agite une cliquette en bronze, $\pi \lambda \alpha \tau \alpha^{\prime} \gamma \eta \chi \alpha \lambda \kappa \varepsilon i ́ n$, pour chasser les oiseaux carnivores du lac Stymphale lors de son sixième travail ${ }^{48}$. Un aphorisme garde le souvenir de cet exploit qui restaure l'ordre dans la région : " Il fit une cliquette en bronze et l'agita. " Cependant, l'expression est uniquement littéraire. Les imagiers grecs ne représentent jamais Héraclès armé d'une cliquette, mais d'une fronde, dans les plus anciennes représentations, puis d'un arc et de flèches ${ }^{49}$.

Plutarque décrit aussi la force protectrice et régénératrice du sistre, également en métal, capable d'apaiser la puissance destructrice du dieu Seth/Typhon. Relevons que l'objet doit être secoué et que son agitation pourrait être mise en relation avec celle qui caractérise aussi les enfants :

"Le sistre indique lui aussi que les êtres doivent être agités (seiesthai) et ne jamais cesser de se mouvoir, qu'il faut pour ainsi dire les réveiller et secouer leur torpeur et leur engourdissement ${ }^{50}$."

45. PLINE, Histoire naturelle, XXVIII, 257; XXXII, 137.

46. Dents et défenses animales enfilées sur un bracelet avec divers éléments sonores (monnaie percée, clochette) : DASEN, Véronique, "Protéger l'enfant : amulettes et crepundia ", dans Gourevitch, Danielle, MoIrin, Anna, RouQuet, Nadine (dir.), Maternité et petite enfance dans l'Antiquité romaine, Catalogue de l'exposition, Bourges, Muséum d'histoire naturelle, 6 novembre 2003-28 mars 2004, Bourges, Éditions de la ville de Bourges, Service d'archéologie municipal, 2003, p. 172-177. Sur les amulettes prévenant d'autres maux, voir dans ce volume HuSQuIN Caroline, "Perceptions et accompagnements de l'atteinte physique chez l'enfant dans l'Antiquité romaine".

47. Par ex. MAY, Roland, ANDRÉ, Jean-Marie et al., Jouer dans l'Antiquité [Catalogue de l'exposition], Marseille, Musée d'Archéologie méditerranéenne, Centre de la Vieille Charité, 22 novembre 1991-16 février 1992, Paris, RMN, 1991, p. 51-52.

48. Apollonios De Rhodes, Argonautiques, 2, 1052-1257 : " Mais, agitant dans ses mains une

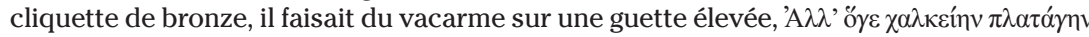

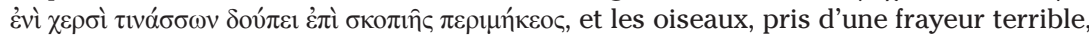
fuyaient au loin en poussant des cris. "Voir aussi DiODORE DE SiCILE, IV, 13, 2, 1-12 (platagê); Apollodore, Bibliothèque, II, 6, 1-12 (krotalon). Huffman, Carl A., Archytas of Tarentum..., op. cit., p. 304-305.

49. Sur l'iconographie de cet épisode, WoOdFord, Susan, s.v. Herakles, dans Lexicon Iconographicum Mythologiae Classicae, Zürich/Munich, V, 1990, p. 54-57, nos 2241 à 2283 , pl. 71-72 (fronde/arc et flèches).

50. Plutarque, Isis et Osiris, 63, Euvres morales 376c-d. Sur le rapport de ce mouvement avec la philosophie atomiste, Kolotourou, Katarina, " Musical Rhythms from the Cradle to the Grave ", dans Haysom, Matthew, Wallensten, Jenny (dir.), Current Approaches 
Dans sa vie de Pythagore, Porphyre explique que le bronze possède une sonorité remarquable qui manifeste la voix d'un daimon:

"Le son produit par un coup sur du bronze était la voix de quelque démon prisonnière de ce bronze ${ }^{51}$."

À l'époque romaine, Columelle recommande l'usage de crepitacula en bronze à des fins plus pragmatiques, effrayer les abeilles pour les obliger à regagner leur ruche ${ }^{52}$.

Les méfaits des oiseaux du lac Stymphale rappellent les attaques d'autres oiseaux carnivores, les striges, qui pénètrent dans les maisons

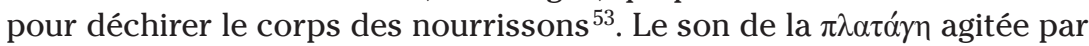
une nourrice avait-il le pouvoir de les chasser? Alexandra Villing met en relation l'invention d'Archytas avec la présence de clochettes et de sonnailles dans les tombes d'enfants ${ }^{54}$. Elle associe ces idiophones au récit de la naissance de Zeus, protégé par le bruit des Courètes frappant leurs armes $^{55}$.

Les hochets conservés ne sont cependant pas tous en métal, mais aussi en terre cuite ou en os. La forme du hochet lui-même a peut-être été conçue pour apaiser les plus petits. Les exemplaires grecs en terre cuite des époques classique et hellénistique présentent une grande variété de types, du porcelet à l'enfant au berceau ou chevauchant un animal ${ }^{56}$.

to Religion in Ancient Greece (Acta Instituti Atheniensis Regni Sueciae, Series in 8, 21), Stockholm, Stockholm Universitet, 2011, p. 180-181.

51. Porphyre, Vie de Pythagore, 41. Sur la sonorité exceptionnelle du bronze, associée à des rites de purification, TuPET, Anne-Marie, La magie dans la poésie latine. Des origines à la fin du règne d'Auguste, Paris, Les Belles Lettres, 1976, p. 39-43; VILLING, Alexandra, "For Whom Did the Bell Toll in Ancient Greece ", The Annual of the British School at Athens, 97, 2002, p. 289-291; Kolotourou, Katarina, " Musical Rhythms... ", art. cité, p. 185-186.

52. COLUMELLE, De l'agriculture, IX, 12, 2.

53. Ovide, Fastes, vi, 132-168; Sorlin, Irène, « Striges et Geloudes. Histoire d'une croyance et d'une tradition ", Travaux et Mémoires. Centre de recherche d'histoire et de civilisation byzantine, 11, 1991, p. 411-436; DASEN Véronique, Le sourire d'Omphale. Maternité et petite enfance dans l'Antiquité, Rennes, PUR, 2015, p. 290-293.

54. Villing, Alexandra, "For Whom Did the Bell Toll... ", art. cité, p. 290.

55. CAllimaque, Hymne à Zeus, 50-52 : "Autour de toi les Courètes menaient leur danse pressée, frappant leurs armes, pour qu'aux oreilles de Cronos vînt le fracas du bouclier, et non pas ton vagissement d'enfant."

56. Bellia, Angela, Strumenti musicali..., op. cit., p. 19-33 (IV siècle av. J.-C. : fig. 30 : cochonnet; fig. 31 : enfant chevauchant un chien; fig. 32 : coq), ép. hellénistique : fig. 33-34 : enfant au berceau). L'auteure relève que certaines sonnailles ne sont probablement pas des hochets pour enfants (p. ex. fig. 26 : grenade, $\mathrm{vI}^{\mathrm{e}}$ siècle av. J.-C.); Voir aussi eadem, "La musica e l'infanzia nel mondo antico ", dans TERRANOVA, Chiara (dir.), La presenza degli Infanti nelle Religioni del Mediterraneo Antico: la vita e la morte, i rituali e $i$ culti tra Archeologia, Antropologia e Storia delle Religioni, Roma, Aracne, 2014, p. 54-71. La fonction des hochets en terre cuite en forme de porcelet et de chouette n'est probablement pas ludique, mais religieuse; BuchHolz, Hans-Günter, "Tönerne Rasseln aus Zypern ", Archäologischer Anzeiger, 2, 1966, p. 140-151; Idem, "Kyprische Eulenrasseln ", Archaeologia Cypria, 2, 1990, p. 33-51; Kolotourou, Katerina, " Music and Cult, the Significance of Percussion and the Cypriote Connection ", dans KaragheOrgis, Vassos, MatTHÄUs, Hartmut, RogGe, Sabine (dir.), Proceedings of an International Symposium 
À l'époque romaine, à côté des chiens et des oiseaux ${ }^{57}$, les exemplaires en forme de visages ou de bustes féminins pourraient avoir évoqué la présence rassurante d'une personne adulte ${ }^{58}$.

\section{Musique et éducation}

La fonction du hochet ne se limite pas à distraire les frayeurs du tout petit pour assurer sa croissance en bonne santé. L'objet participe à un véritable programme éducatif qui vise à introduire l'apprentissage de la raison par le rythme. La musique va faire de l'enfant un petit humain, distinct des animaux. Aristote préconise d'agir sans tarder, car les bonnes ou mauvaises habitudes s'implantent très tôt chez l'enfant de manière indélébile ${ }^{59}$. Le bercement représente en soi une sorte de premier entraînement physique qui structure l'âme :

«Pour former dans l'âme cette vertu particulière [le courage], les mouvements qu'on imprime ainsi aux tout petits constituent donc, disons-le, une gymnastique grandement utile ${ }^{60}$."

Les auteurs d'époque romaine relaient ces principes. Au II $^{\mathrm{e}}$ siècle apr. J.-C., Plutarque compare la malléabilité de l'âme du jeune enfant, paidion, à de la cire, marquée de manière indélébile par les premières expériences comme d'un sceau, en citant le poète Phocylide (vI ${ }^{\mathrm{e}}$ siècle av. J.-C.) qui recommande instamment d'enseigner "à bien faire " dès l'enfance ${ }^{61}$.

L'apprentissage du rythme, eurythmia, de la musique et de la gymnastique forme ainsi la base de l'éducation dans le but d'atteindre l'harmonie du corps et de l'âme. L'éducation est d'autant plus importante que la vitalité extraordinaire des plus jeunes est associée à l'absence de contrôle de leurs émotions. Platon relève qu'il est indispensable de leur faire acquérir une discipline le plus tôt possible :

on Cypriote Archaeology, Erlangen, 23-24 July 2004, Möhnesee-Wamel, Bibliopolis, 2005, p. 186-187.

57. P. ex. PITARAKIS, Brigitte, " The Material Culture of Childhood in Byzantium ", dans Papaconstantinou, Arietta, Talbot, Alice-Mary (dir.), Becoming Byzantine. Children and Childhood in Byzantium, Washington, D.C., Dumbarton Oaks Research Library and Collection, 2009, p. 219-222, fig. 18-19 (chien et coq); Messineo, Gaetano, "Puerilia crepitacula?", Rivista di Studi Pompeiani, 5, 1991-1992, p. 119-132 (porcelet, buste féminin); PFÄFfL, Barbara, " Rasseln für Kinder aus Augusta Raurica ", Jahresberichte aus Augst und Kaiseraugst, 35, 2014, p. 141-156 (coq, objets imitant l'osier).

58. Par ex. Jouer dans l'Antiquité, 1991, p. 51, fig. 11 (cucullatus); DASEN, Véronique, Le sourire d'Omphale..., op. cit., p. 127, fig. 4.7 (dea nutrix ou nourrice allaitant un enfant).

59. ARISTOTE, Éthique à Nicomaque, II, 1-2, 1103b, 23-25 : "L'importance de contracter telle ou telle habitude dès la prime jeunesse n'est donc pas négligeable, mais tout à fait décisive."

60. Platon, Lois, VII, 791 c.

61. Plutarque, De l'éducation des enfants, 5f, Euvres morales 3d : " Et il y a toute chance que le poète Phocylide donne un bon conseil quand il dit : "Il faudrait, dès l'enfance,

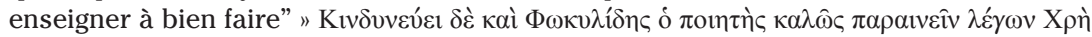

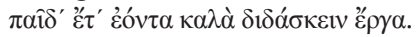


" Tout ce que je sais, dit l'Athénien, c'est qu'absolument aucun être ne possède jamais, quand il vient au monde, toute la somme de raison que doit avoir son âge adulte; or, dans le temps où il n'a pas encore son compte d'intelligence, chacun est fou et crie sans règle, et dès qu'il se dresse sur ses pieds, sans règle encore il se met à sauter."

Si tous les êtres jeunes sont caractérisés par des mouvements et des cris désordonnés, ce qui distingue l'être humain de l'animal est le plaisir qu'il prend au rythme. L'acquisition du sens du rythme l'initie à celui de l'harmonie, et par là le mène à un esprit rationnel.

"Tous les êtres jeunes, ou à peu près, sont incapables de tenir en repos leur corps et leur voix; ils cherchent sans cesse à remuer et à parler, les uns en sautant et bondissant, comme s'ils dansaient de plaisir et jouaient entre eux, les autres en émettant tous les sons de voix possibles. Or les animaux n'ont pas le sens de l'ordre et du désordre dans leurs mouvements, de ce qu'on appelle rythme et harmonie; mais à nous, les dieux [...] ont donné un sens du rythme et de l'harmonie accompagné de plaisir, par lequel ils nous mettent en branle en se faisant nos chorèges; et ils ont appelé cela les chœurs, du nom de la joie qu'on y ressent ${ }^{62}$."

Si le bercement peut être considéré comme une première forme de gymnastique, le hochet représente une initiation au rythme et à la musique, à laquelle "les petits enfants aussitôt nés prennent plaisir ", relève PseudoAristote $^{63}$, car elle structure le corps et l'esprit :

"Un rythme nous plaît parce qu'il a un nombre familier et régulier, et qu'il nous entraîne d'une manière ordonnée ${ }^{64}$."

Ces préceptes rejoignent les recommandations des Pythagoriciens sur la valeur éducative de la musique. D'origine divine, la musique transmet l'harmonie cosmique ${ }^{65}$. Dans le Cratyle, Platon explique les liens qui unissent les compétences guérisseuses et musicales d'Apollon, le dieu qui délivre à la fois les maux du corps et de l'âme ${ }^{66}$. Dans ce cercle, Archytas de Tarente, proche de Pythagore, est un pionnier renommé de la théorie musicale. Le savant aurait eu un intérêt particulier pour les instruments idiophones de la famille de la platagê, et aurait établi une théorie sur les vibrations sonores et la propagation des sons. Un fragment de ce traité, cité par Porphyre, examine les variations de l'intensité sonore selon la force ou puissance du choc sonore, en décrivant comment se produisent les sons ${ }^{67}$. L'invention qu'Aristote lui attribue peut ainsi être comprise dans le contexte pédagogique plus large d'une formation de l'enfant au moyen d'un apprentissage actif, tel qu'il le prône :

62. Platon, Lois, II, 653b-654a. Voir aussi Platon, Protagoras, 326 b (sur la nécessité de

l'eurythmia et l'euarmostia); Kolotourou Katerina, " Musical Rhythms... ", art. cité, p. 180.

63. Pseudo-Aristote, Problèmes, xix, 38, $902 \mathrm{~b}$.

64. Ibidem.

65. StRABOn, X, 3, 10.

66. Platon, Cratyle, 405a-d.

67. Porphyre, Sur les harmoniques de Ptolémée, I, 3; Huffman, Carl A., Archytas of Tarentum, op. cit., Fragment 1, L. 7-43 (A, B, p. 103-108, 129-148). 
"Les enfants doivent-ils ou non apprendre la musique en chantant et en jouant eux-mêmes? Il est bien évident que, pour acquérir telle ou telle disposition, la participation personnelle à l'exécution a une grande importance; il est, en effet, impossible ou difficile de devenir bon juge d'activités auxquelles on n'a pas pris part ${ }^{68}$."

La fin du passage d'Aristote, souvent coupée, confirme l'intention pédagogique dans laquelle s'inscrit probablement le concept d'Archytas :

" Il faut considérer comme une belle invention le hochet, platagê, d'Archytas que l'on donne aux petits enfants pour que, grâce à elle, ils ne cassent rien dans la maison, car la gent enfantine n'est pas capable de rester tranquille. Le hochet convient donc bien aux enfants en bas âge, et l'éducation, c'est un hochet, platagê, pour les jeunes qui ont grandi ${ }^{69}$."

"Ne rien casser dans la maison " n'est donc pas une recommandation légère. Le hochet permet de canaliser l'énergie enfantine en offrant à l'enfant une activité " bien réglée et de bon équilibre ", en exerçant une motricité fine, et en l'empêchant de prendre de mauvaises habitudes. La préoccupation est centrale chez Platon, articulée au souci de procurer très tôt une formation musicale :

"Le maître de cithare doit se préoccuper [...] d'empêcher la jeunesse de se conduire mal en quoi que ce soit [...] en ayant appris à jouer la cithare et à chanter il force ainsi le rythme et l'harmonie à devenir familiers à l'âme des enfants afin de rendre ceux-ci plus heureusement réglés dans leurs mouvements ${ }^{70}$."

Le hochet d'Archytas représente ainsi un moyen idéal d'initiation à la musique, favorisé par le plaisir qu'y prend l'enfant :

"L'enseignement de la musique est bien adapté à la nature de la jeunesse : les jeunes, à cause de leur âge, ne supportent jamais volontiers ce qui n'a pas d'agrément, et la musique par nature fait partie des choses qui ont une agréable douceur. Et il paraît y avoir en nous quelque affinité avec les harmonies et les rythmes ${ }^{71}$."

Cette fonction formatrice se retrouve à l'époque romaine dans la description du nouveau-né dans le poème De la nature. Lucrèce fait du jouet l'accessoire qui distingue l'homme de l'animal, révélant l'état de dépendance physique et psychique du petit humain à la naissance, ainsi que l'importance de ses interactions avec les adultes qui vont en faire un être social $^{72}$ :

"Pareil au naufragé que les flots ont rejeté sur le rivage, le nouveau-né gît, tout nu, par terre, incapable de parler, dépourvu de tout ce qui aide à

68. ARIStote, Politique, VIII, 6, 3, 1340b : «Ainsi donc, la musique doit s'enseigner d'une façon pratique par des exercices auxquels on prend part, comme le montrent clairement les considérations précédentes."

69. ARISTOTE, Politique, viII, 6, 2, $1340 \mathrm{~b}$.

70. Platon, Protagoras, 326a-b.

71. ARISTOTE, Politique, VIII, 5, 25, 1340b.

72. LuCRÈCE, De la nature, v, 229-230. 
vivre, arraché avec effort du ventre de sa mère. [...] Heureux les animaux domestiques, gros et petits, et les bêtes sauvages : ils n'ont besoin ni de hochets, crepitacilla, ni des mots caressants que chuchote la voix d'une tendre nourrice. "

À l'inverse, les animaux se débrouillent de manière autonome dès le début :

"Ils ne sont pas en quête de vêtements qui changent avec les époques de l'année, ils n'ont besoin ni d'armes ni de hautes murailles pour défendre leurs biens ", puisque " la terre et la nature inventive enfantent d'elles-mêmes toutes sortes d'abondantes ressources afin de veiller à leurs besoins ${ }^{73}$."

\section{L'invention d'Archytas}

L'invention d'Archytas pourrait ainsi résider non dans la découverte de l'objet, qui existait depuis longtemps, sous différentes formes et en divers matériaux, mais dans la mise au point d'un concept éducatif qui pourrait avoir représenté l'application pratique de ses réflexions théoriques sur le pouvoir du son, du rythme et de la musique.

Plusieurs anecdotes signalent l'intérêt particulier qu'Archytas portait aux enfants. Selon Élien (II ${ }^{\mathrm{e}}$ siècle apr. J.-C.), il favorisait leur compagnie ${ }^{74}$ :

"Même Archytas de Tarente, qui fut à la fois politicien et philosophe et qui possédait de nombreux esclaves, prenait un vif plaisir avec leurs enfants et jouait avec ceux qui étaient nés dans sa maison. Il aimait surtout à s'amuser avec eux dans les banquets."

Son observation attentive de leurs comportements et de leurs jeux a pu le conduire à élaborer une théorie sur la valeur éducative de la platagê. "Le hochet d'Archytas " ferait ainsi partie d'un programme d'anthropopoiêsis, de transformation du petit humain en être social, par un premier apprentissage du rythme et du son qui structure son corps et son âme. Le proverbe fait aussi référence à l'agitation permanente que les enfants doivent apprendre à maîtriser en grandissant.

Relevons que l'inspiration d'Archytas puise dans un fond plus ancien. Les instruments idiophones par entrechoc ou secouement accompagnent la croissance des enfants grecs depuis l'époque archaïque. Leur forme se transforme selon l'âge. La platagê des plus petits devient les crotales ou platagêma associées aux chants et danses des jeunes gens. Dans un poème de

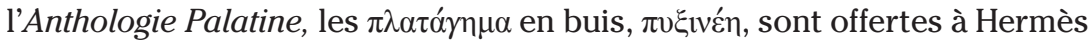
à la sortie de l'enfance d'un garçon. Elles sont associées à des jeux qualifiés de paignia qui procurent du plaisir :

"Philoclès a consacré à Hermès son ballon (sphaira) renommé, ces crotales (platagêma) de buis, les osselets (astragaloi) qu'il a aimés à la folie,

73. Ibidem.

74. ÉLIEN, Histoire variée, XII, 15. Voir aussi ATHÉNÉE, Les Deipnosophistes, XII, 519 b; Huffman, Carl A., Archytas of Tarentum..., op. cit., p. 297-302, A8, A10. 
et le rhombe (rhombos) qu'il faisait tournoyer : tous les jouets (paignia) de son enfance ${ }^{75}$."

\section{Des hochets et des hommes}

Loin d'être un objet anodin, le hochet, platagê, témoigne ainsi de l'attention portée aux enfants dès leur plus jeune âge. Il s'inscrit dans un ensemble plus large de soins, en partie immatériels, dont les berceuses et les comptines font aussi partie. Sa fonction est prévue pour assurer le bien-être à la fois physique et mental du tout petit, et renvoie à une façon de penser le corps de l'enfant, sa santé et son développement harmonieux.

L'imagerie grecque confirme que l'idiophone n'était pas uniquement utilisé pour endormir les plus petits. Le programme éducatif contenu dans l'expression "le hochet d'Archytas " est mis en œuvre sur la scène du chous conservé à Londres (fig. 1). Le peintre a réuni autour de l'enfant, un garçon, un ensemble d'objets spécifiques à sa catégorie d'âge et à son sexe qui construisent un discours visuel sur le processus d'apprentissage du futur citoyen : le petit est assis sur un pot, lasanon, attesté archéologiquement ${ }^{76}$, qui signale l'acquisition du contrôle de son corps, du propre et du sale $^{77}$. À ses côtés, le bâton à roulettes fait référence à l'apprentissage de la marche, peut-être aussi aux cadeaux que recevaient les enfants à l'occasion des fêtes de la cité ${ }^{78}$, tandis que le cruchon à vin renvoie à une première participation à la fête dionysiaque des Anthesthéries, et plus largement à la vie religieuse communautaire ${ }^{79}$. Le vin contenu dans le chous représente également la boisson des enfants qui grandissent. Le traité hippocratique Du régime salutaire (fin $\mathrm{V}^{\mathrm{e}}$ siècle av. J.-C.) le recommande, mais coupé d'eau " et non tout à fait froid ", avec les qualités appropriées, " qui ne gonfle pas le ventre ni ne cause de flatuosités", afin que les petits "soient moins sujets aux convulsions et qu'ils aient plus d'embonpoint et de couleur ${ }^{80}$ ".

75. LÉONIDAS de Tarente, Anthologie Palatine, vI, 309.

76. Lynch, Kathleen M., PAPAdOPOUlos, John K., "Sella Cacatoria : A Study of the Potty in Archaic and Classical Athens ", Hesperia, 75, 2006, p. 1-32; VILLING Alexandra, dans Stampolidis, Nicholas Chr., Tassoulas, Yorgos (dir.), Hygieia. Health, Illness, Treatment from Homer to Galen, Athènes, Museum of Cycladic Art, 2014, nº 16, p. 120-121.

77. Sur l'observation du méconium et des selles des bébés, ARISTOTE, Histoire des animaux, VII, 10, 587a 28-33, et VII, 11, 587b 7-8.

78. Cf. ARISTOPHANE, Les Nuées, 863-864 : "La première obole que je reçus comme héliaste, je l'employai à t'acheter, aux Diasia, un petit chariot "; BEAUMONT, Leslie A., Childhood in Ancient Athens..., op. cit., p. 78.

79. NEILS, Jennifer, "Children and Greek Religion ", dans NEILS, Jennifer, OAKLEY, John (dir.), Coming of Age in Ancient Greece..., op. cit., p. 145-147.

80. HiPPOCRATE, Du régime salutaire, 6 (Littré, vI, 80-83). Sur la prescription de vin coupé d'eau pour les enfants, voir aussi par ex. Hippocrate, Des airs, des eaux et des lieux, IX (Littré, II, 40-41). ARISTOTE, Politique, VII, 16, 1, 1335b et Histoire des animaux, VII, 12, 588a, 3-10 déconseille cependant de donner du vin aux enfants, directement ou par le lait de la nourrice, car il peut causer les convulsions redoutées. Sur l'usage probatoire du vin à Sparte, voir aussi dans ce volume DAMET, Aurélie, " De Sparte à l'île du Soleil "; sur le rôle qu'on lui attribuait dans l'épilepsie et les convulsions, voir aussi dans ce volume 
La présence du hochet dans la main de l'enfant complète le tableau. Sa manipulation renvoie à la formation de l'âme et de la raison en train de s'opérer par le rythme de la musique. Si l'enfant est représenté seul, sans adulte ni compagnon, tout parle sur le chous de ses proches et de l'attention dont il est l'objet. Sa gestualité suggère aussi un apprentissage social par l'interaction : il tend le bras vers l'extérieur de la scène, en dirigeant son regard vers celles ou ceux que l'imagier ne montre pas, mais dont la présence est rendue implicite, la mère, la nourrice ou le pédagogue. Deux vases dépeignent une scène similaire où l'enfant est assis sur son pot, sans hochet cette fois, mais en présence de la mère, imitant ses gestes ou tendant les bras vers elle ${ }^{81}$.

La diversité typologique des hochets conservés en métal, os et terre cuite ne correspond pas aux rares représentations de l'objet ${ }^{82}$. Sur les vases attiques, aucun idiophone n'a de forme animale. Le type le plus commun ressemble à une sorte de gourde, comme sur le chous du British Museum (fig. 1); le rehaut blanc pourrait indiquer ici un matériau métallique. Ailleurs l'identification est parfois incertaine ${ }^{83}$. Sur un chous conservé au Musée national d'Athènes (fig. 2 ; vers 400-390 av. J.-C.) ${ }^{84}$, une fillette debout, vêtue d'une tunique, tient le manche d'un objet de forme rectangulaire qui évoque les plaquettes en os découvertes dans des tombes d'enfant en Sicile (IV ${ }^{\mathrm{e}}$ siècle av. J.-C.) ${ }^{85}$. Elle l'agite devant un garçonnet nu agenouillé devant un tabouret sur lequel il s'appuie. Elle tient de l'autre main un gâteau, alliant ainsi l'invitation à deux plaisirs, ceux de la musique et de la nourriture.

GAILLARD-SEuX, Patricia, "L'épilepsie de l'enfant dans l'Antiquité ( ${ }^{\mathrm{er}}-\mathrm{V}^{\mathrm{e}}$ siècles) : prévention et traitement".

81. Scènes similaires : coupe attique à fond blanc, médaillon à figures rouges (vers 460 av. J.-C.), Bruxelles, musées royaux d'Art et d'Histoire A 890, ARV² 771, 1, Add ${ }^{2} 287$; BA 209536 (P. de Sotades); lécythe attique à figures rouges (475-460 av. J.-C.), Berlin, Antikensammlung, Staatliche Museen F 2209; ARV² 1587.2; CreLIER, Marie-Claire, Kinder in Athen im gesellschaftlichen Wandel des 5. Jahrhunderts v. Chr. Eine archäologische Annäherung, Remshalden 2008, K1 B et L7 (avec illustrations).

82. Quelques chercheurs ont proposé d'identifier le " hochet d'Archytas " avec une échelette telle que figurée sur des vases d'Italie du sud du Iv e siècle av. J.-C.; SMITH, Henry W.R., Funerary Symbolism in Apulian Vase Painting, Berkeley, University of California Press, 1976, p. 129-132. Cette interprétation est aujourd'hui rejetée : HufFmAn, Carl A., Archytas of Tarentum..., op. cit., p. 305-306.

83. L'identification est parfois débattue. Autres hochets, de forme allongée ou ovoïde : VAN HoORn, Gerard, Choes and Anthesteria..., op. cit., n 239, fig. 293 (de l'Agora d'Athènes), no 551, fig. 291, et no 925, fig. 399 (d'une nécropole de Tarente). Cf. HatzivassiLIou, Eleni, "The Attic Phormiskos : Problems of Origin and Function ", Bulletin of the Institute of Classical Studies, 45, 2001, p. 113-148.

84. De Rhamnonte. VAN HOORN, Gerard, Choes and Anthesteria..., op. cit., $\mathrm{n}^{\circ}$ 38, fig. 295. Sur deux stèles funéraires attiques $d_{u} \mathrm{IV}^{\mathrm{e}} \mathrm{s}$. des enfants tiennent dans la main le même genre de platagê rectangulaire; Clairmont, Christophe W., Classical Attic Tombstones, Kilchberg, Akanthus, 1993, 0.722 (jeune fille, Baltimore, The Walters Art Gallery) et 0.845 (garçon, Athènes, Musée national).

85. Plaquettes rectangulaires en os, de Monte Sannace (région de Tarente) et Rugge (long. 12 cm, larg. 2,4-3,2 cm); Bellia, Angela, Strumenti musicali..., op. cit., p. 15-16, fig. 14-16. 
L'apprentissage du rythme est associé à celui de la danse sur un chous conservé à Munich (fig. 3; vers 440-400 av. J.-C.) ${ }^{86}$. Un garçonnet, nu, au corps potelé, s'avance en tenant de la main droite un hochet rectangulaire. Deux oiseaux, peut-être des canards, l'entourent, évoquant la parenté du monde animal et de l'enfance. Les trois figures semblent avancer au rythme du hochet, à la fois proches et déjà différentes.

Figure 1 - Chous attique (H. 10,5 cm). Londres, British Museum 1910.615.4. () The Trustees of the British Museum.

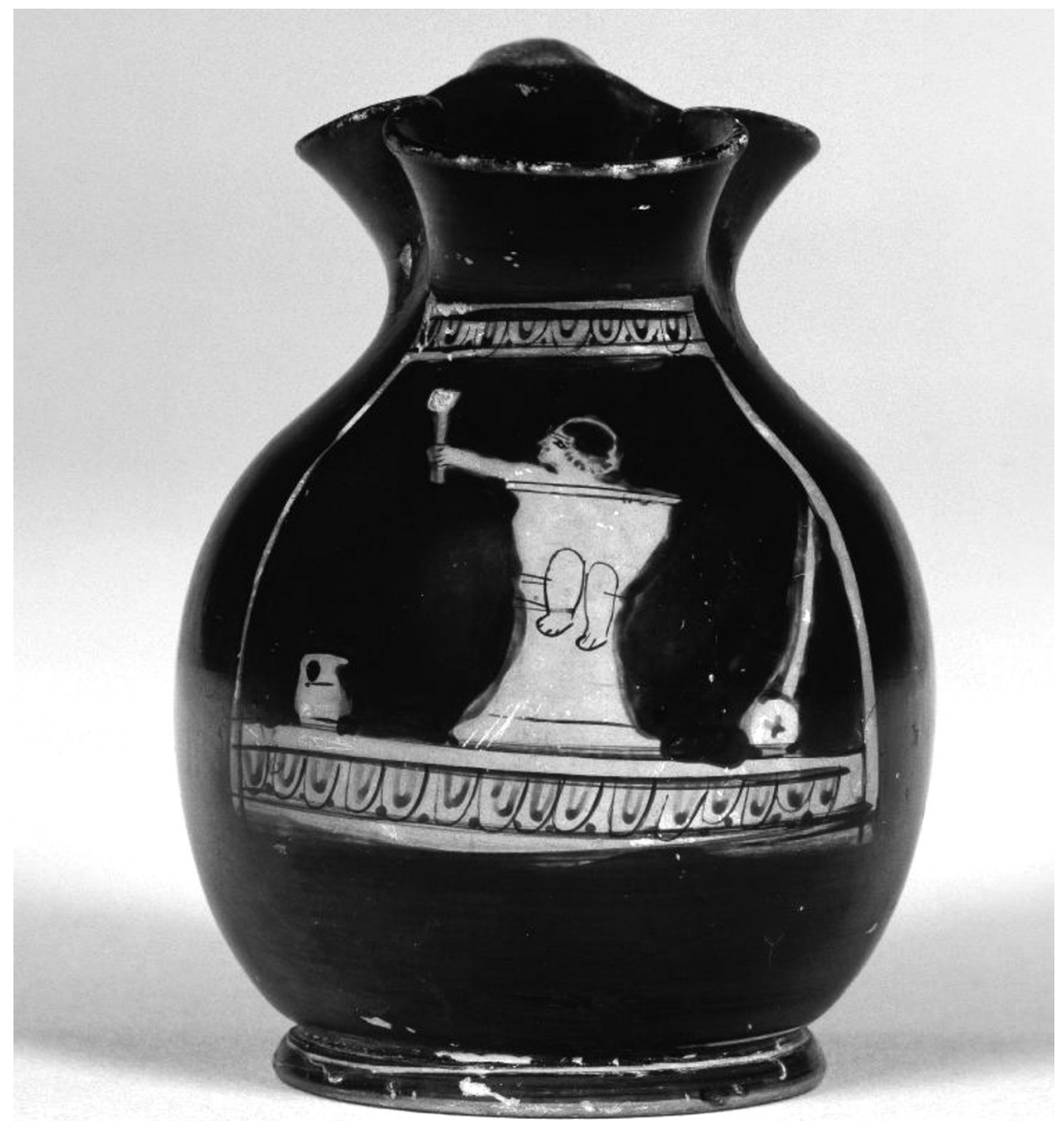

86. Munich Antikensammlung 2462. vAN HooRn, Gerard, Choes and Anthesteria..., op. cit., fig. 294. 
Figure 2 - Chous attique $(H .8 \mathrm{~cm})$. Athènes, Musée national, Inv. 1268-CC 1878. Photo V. Martini.
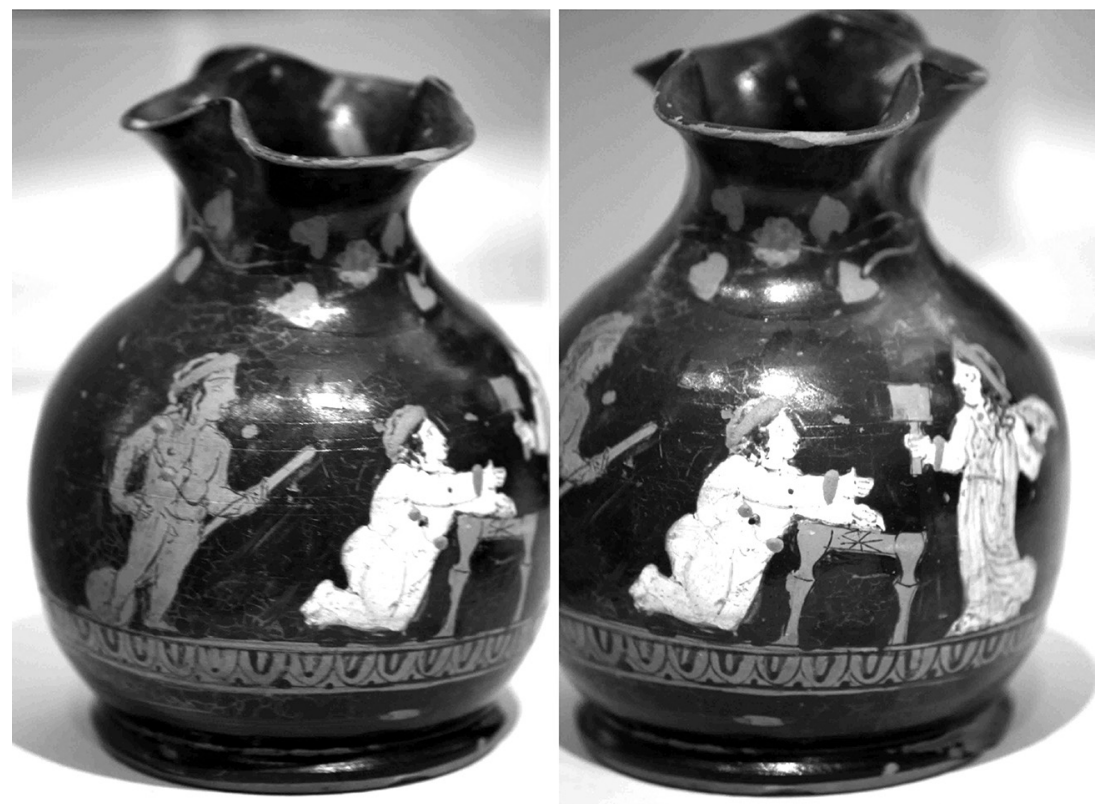

Figure 3 - Chous attique $(H .7 \mathrm{~cm})$. Munich, Antikensammlungen, Museum antiker Kleinkunst 2462. Dessin V. Dasen.

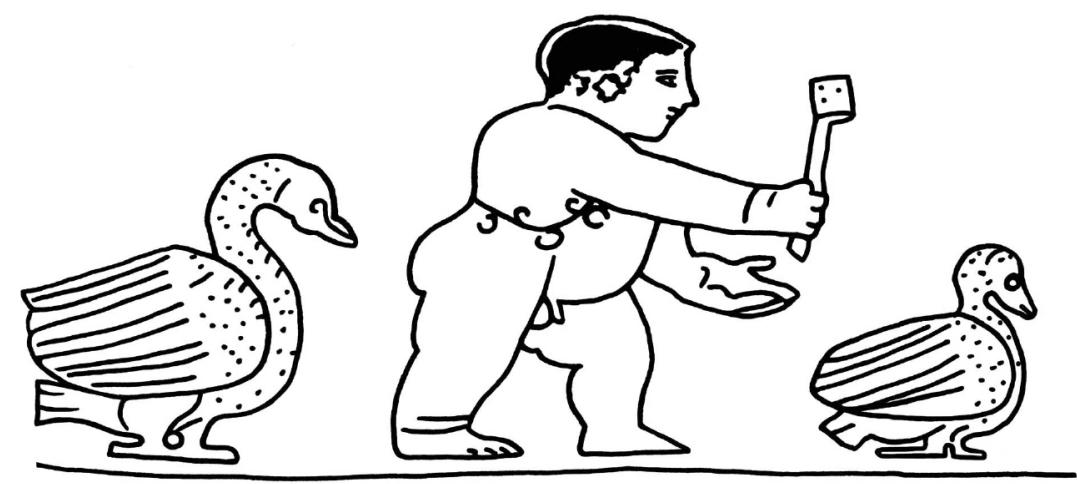




\section{Abréviations}

BA : Beazley Archive [http://www.beazley.ox.ac.uk/pottery/default.htm].

Dar. : ORIBASE, Euvres. Texte et traduction par V. Bussemaker et Ch. Daremberg, Paris, 1851-1876, 6 vol.

Littré : É. Littré, CEuvres complètes d'Hippocrate. Traduction nouvelle avec le texte grec en regard, Paris, 1839-1861, 10 vol.

Kühn : C.G. Kühn (éd. et trad. lat.), Galeni Opera omnia, Leipzig, 1821-1833, 20 vol. (réimpr. Hildesheim 1964-1965).

\section{RÉSUMÉ}

Aristote attribue à Archytas de Tarente l'invention du hochet. La découverte ne concerne cependant pas l'objet, mais le concept qui détermine son usage. Elle prend tout son sens à la lumière de la dimension éducative de la musique prônée par le pythagoricien et ses contemporains. Loin d'être un simple objet ludique, "le hochet d'Archytas " participe pleinement au processus de " fabrication de l'humain " qui transforme par étapes le tout-petit en être social en Grèce ancienne. Le rythme et la musique structurent l'âme de l'enfant, tout en le détournant d'activités désordonnées qui font prendre de mauvaises habitudes. Son usage témoigne du souci d'entourer le tout petit de soins adaptés à son âge dès la naissance. Il s'inscrit aussi dans une gamme de soins qui inclut les berceuses et visent à assurer une croissance en bonne santé. La représentation sur un chous attique d'un enfant assis sur son pot, entouré d'un bâton à roulettes, d'un chous et brandissant un hochet résume ce programme d'anthropopoiêsis : il associe l'apprentissage de la propreté, de la marche, des rites religieux de la cité, à la formation de l'âme par le rythme musical du hochet.

\section{ABSTRACT}

Aristotle attributes to Archytas of Tarentum the invention of the rattle. However, the discovery does not concern the object, but the concept which determines its use, as the educational dimension of music advocated by the Pythagorean and his contemporaries reveals. Far from being a simple playful object, the rattle fully participates in the process of "making human" which transforms step by step the toddler into a social being in ancient Greece. Rhythm and music structure the mind of the child, also diverting him away from activities that cause bad habits. Its use demonstrates the desire to surround the child with care adapted to his/her age from birth. It is also part of a range of care, including lullabies, that aim at ensuring a healthy growth. The depiction on an Attic chous of a child sitting on a pot beside a wheel cart and a chous, holding a rattle, epitomises this program of anthropoiesis: the learning of cleanliness, walking, community religious rites, and the training of the soul by the musical rhythm of the rattle. 